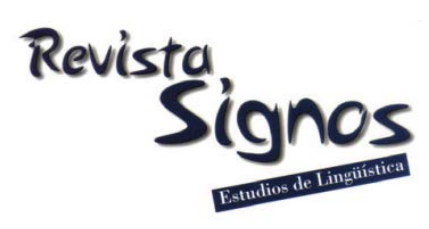

\title{
Habilidades favorecedoras del aprendizaje de la lectura en alumnos de 5 y 6 años
}

\author{
Raúl Gutiérrez \\ UNIVERSIDAD DE ALICANTE \\ ESPAÑA \\ raul.gutierrez@ua.es
}

Favoring skills of learning to read in 5 and 6 years students

Recibido: 24-IX-2016 / Aceptado: 17-IV-2017

\section{Resumen}

El interés por el aprendizaje de la lectura, así como por la capacidad de favorecer la adquisición de esta habilidad lingüística en las primeras edades ha sido objeto de numerosas investigaciones en los últimos años. Desde este marco, el objetivo de este estudio fue analizar el efecto que la intervención conjunta en conciencia fonológica, velocidad de denominación y conocimiento alfabético tiene sobre el aprendizaje de la lectura durante el proceso de adquisición de esta habilidad lingüística. Se empleó un diseño cuasi-experimental de comparación entre grupos con medidas pretest y postest. Participaron 408 alumnos con edades comprendidas entre los 5 y los 6 años. El grupo experimental obtuvo puntuaciones significativamente más altas que el grupo control en las pruebas de conciencia fonológica, denominación rápida y conocimiento del código escrito logrando niveles superiores en las tareas de lectura. Los resultados apoyan el desarrollo de modelos didácticos que integren estas variables tanto para la mejora del aprendizaje lector en las primeras edades, como para la prevención de dificultades en esta habilidad lingüística, lo que presenta importantes implicaciones educativas para la enseñanza de la lectura en el aula.

Palabras Clave: Lectura, conciencia fonológica, velocidad denominación, comprensión, lenguaje escrito. 


\begin{abstract}
Interest in learning to read as well as the ability to predict the acquisition of this linguistic ability in early age has been the subject of numerous investigations in recent years. In the context, the aim of this study was to analyze the effect that the joint intervention in phonemic awareness, alphabetic naming speed and is knowledge about learning reading during the acquisition process of this linguistic skill. A quasiexperimental design compared between groups with pretest posttest measures and was employed. Participants 408 students aged 5 to 6 years. The experimental group had significantly higher scores than the control tests phonological awareness, rapid naming and knowledge of the written code group achieving higher levels in reading assignments. The results support the development of educational models that integrate these variables for both improving learning reader in the early ages, and for the prevention of difficulties in this linguistic ability, which has important educational implications for reading instruction in the classroom.
\end{abstract}

Key Words: Reading, phonological awareness, naming speed, comprehension, written language.

\title{
INTRODUCCIÓN
}

Para aprender a leer en un sistema alfabético, como el castellano, se requiere del conocimiento de la relación entre el lenguaje oral y escrito, debido a que nuestro sistema de representación se basa en la segmentación de la cadena del habla. Cuando los niños se inician en el proceso de adquisición de la lectura han de saber que las letras son signos gráficos que se corresponden con los elementos sonoros y que cada letra se asocia con una unidad de sonido. La adquisición de esta relación facilita el aprendizaje de los procesos de correspondencia grafema/fonema, lo que posibilita que los aprendices puedan deducir los sonidos de las letras a partir de los nombres de las mismas (González, López, Vilar \& Rodríguez, 2013). Este logro requiere el desarrollo de habilidades fonológicas puesto que son éstas las que facilitan la reflexión y la capacidad de manipular las unidades de las palabras del lenguaje hablado. A lo largo de las últimas décadas, distintos estudios han demostrado el papel crucial de las habilidades de conciencia fonológica en la adquisición y en el desarrollo de la lectura, así como en la explicitación de sus dificultades (Arancibia, Bizama \& Sáez, 2012; Gutiérrez, 2016a).

La conciencia fonológica se refiere a la habilidad para identificar, segmentar o combinar, de manera intencional, las palabras que componen las oraciones (conciencia léxica), las sílabas (conciencia silábica), los elementos intrasilábicos (conciencia intrasilábica) así como los fonemas (conciencia fonémica). De todos estos niveles de conciencia fonológica, actualmente existe acuerdo en que la capacidad para manejar las unidades mínimas de las palabras y descubrir la secuencia de los fonemas que las componen, es el aspecto que más relación presenta con el aprendizaje de la lectura debido a que cuanto mejor se identifiquen los fonemas de una palabra mayor facilidad 
existirá para asociar los sonidos con sus correspondientes grafemas (Arancibia, et al., 2012; Suárez-Coalla, García de Castro \& Cuetos, 2013).

Junto con la conciencia fonológica se ha demostrado que la velocidad de denominación presenta una importante influencia en la adquisición de la lectura, en cuanto que determina la rapidez con la que puede ser recuperada una información fonológica almacenada en la memoria a largo plazo y porque permite acceder a las representaciones ortográficas de las palabras (Wolf, O'Rourke, Gidney, Lovett, Cirino \& Morris, 2002). La realización de tareas de velocidad de denominación es predictora de los logros en el aprendizaje de la lectura en las ortografías transparentes, aunque también lo es, en menor grado, en las opacas (Arancibia, et al., 2012; LópezEscribano, Sánchez-Hípola, Suro \& Leal, 2014; Rabazo, García \& Sánchez, 2016).

La fuerte vinculación de la denominación de letras con la fluidez de la lectura de palabras en castellano puede ser atribuible al hecho de que los nombres de las letras en esta lengua proporcionan información clara y consistente de los sonidos que les corresponden, lo que constituye el aspecto fundamental de la decodificación de las unidades lingüísticas del alfabeto (Kim \& Pallante, 2012).

A pesar de la influencia tanto del procesamiento fonológico como de la velocidad de denominación en el aprendizaje de la lectura se ha demostrado que cada una de estas variables interviene de forma diferente en los niveles léxico y subléxico, implicados en la lectura de palabras. Así mientras que el procesamiento fonológico tiene una mayor relación con la exactitud lectora, la velocidad de denominación se encuentra relacionada fundamentalmente con la fluidez lectora (Suárez-Coalla, et al., 2013). Lo que indica que los niños con una menor rapidez para identificar y recuperar la representación fonológica de las palabras o letras serían lectores más lentos.

El conocimiento del nombre de las letras también se ha investigado como un componente importante del proceso temprano de alfabetización, evidenciándose que es un factor de gran relevancia en el aprendizaje del lenguaje escrito (Diuk \& Ferroni, 2012). El conocimiento alfabético facilita el desarrollo de habilidades fonológicas al establecerse una relación causal entre el conocimiento del nombre de las letras y el aprendizaje de sus sonidos, así se ha evidenciado en estudios longitudinales y de correlación realizados en diferentes lenguas (De Jong \& Van der Leij, 2002; LópezEscribano \& Beltrán, 2009).

Ahora bien, un problema de la mayor parte de los estudios destinados a analizar las habilidades que intervienen en el aprendizaje lector (conciencia fonológica, velocidad de denominación y conocimiento alfabético), es que son de tipo correlacional, es decir, se ha intentado demostrar que los niños con mejores resultados en velocidad de denominación y conciencia fonológica son los que mayores niveles de lectura presentan (Martínez, 2014; Gómez-Velázquez, González-Garrido, Matute \& 
Ramos-Loyo, 2014). Sin embargo, estos trabajos no ofrecen una explicación de dichas correlaciones, pudiendo deberse estos resultados a otras variables no analizadas. El mejor modo de comprobar si existe una relación causa-efecto es interviniendo sobre los predictores de la lectura ya que así podremos conocer si la mejoría alcanzada está asociada a la intervención efectuada. Otra limitación que se encuentra en los trabajos conocidos es la intervención parcial sobre los predictores señalados, en unos casos se actúa sobre la conciencia fonológica, en otros sobre la velocidad de denominación, y en otros sobre ambos, normalmente cuando los alumnos ya saben leer, pero no se ha planteado una intervención conjunta sobre los tres predictores de la lectura (LópezEscribano, et al., 2014; Rabazo, et al., 2016).

El objetivo de este estudio fue analizar el efecto que la intervención conjunta en conciencia fonológica, velocidad de denominación y conocimiento alfabético presenta sobre el aprendizaje de la lectura. Para ello se compara el aprendizaje de la lectura de dos muestras de niños de entre 5 y 6 años, uno que recibe instrucción sobre estas variables y otro que sigue el curso normal de escolaridad, analizándose igualmente si existen diferencias en función del género. Nuestra hipótesis es que los alumnos que participan en el programa de intervención mejorarán su proceso lector como consecuencia del desarrollo de la conciencia fonológica, la velocidad de denominación y el conocimiento alfabético y no se producirán diferencias respecto al género del alumnado.

\section{Método}

\subsection{Muestra}

Para la selección de los participantes se tuvieron en cuenta tanto la heterogeneidad de los centros (público y concertado) como el hecho de que estos estuvieran ubicados en un contexto sociocultural de nivel medio. La muestra estaba compuesta por 408 alumnos de primer curso de Educación Primaria con edades comprendidas entre los 5 y los 6 años (Media $=5.36$; DT $=0.45$ ), de los cuales el 49,6\% eran niños y el 50,4\% niñas. Estos alumnos pertenecían a cuatro centros educativos públicos y concertados, de los cuales dos colegios fueron asignados al grupo experimental (206 alumnos) y los otros 2 al grupo control (202 alumnos). De los 206 participantes experimentales, el $50,5 \%$ son varones y el 49,5\% mujeres, mientras que de los 202 participantes del grupo control, el 48,3\% son varones y el $51,7 \%$ son mujeres. El análisis de contingencia (chi cuadrado de Pearson) entre condición y sexo no evidenció diferencias estadísticamente significativas $\left(\mathrm{X}^{2}=0.64, p>.05\right)$.

\subsection{Instrumentos}

Con la finalidad de evaluar las variables dependientes objeto de estudio se utilizaron cuatro instrumentos de evaluación con garantías psicométricas de fiabilidad 
y validez para valorar la conciencia fonológica, la velocidad de denominación y el dominio del proceso decodificador.

- Prueba para la Evaluación del Conocimiento Fonológico (PECO) (Ramos \& Cuadrado, 2006): Este test evalúa los niveles de conocimiento fonológico (silábico y fonémico), cada uno de los cuales se compone de tres tareas distintas; identificación, adición y omisión. Esta prueba incluye tres subtests con sílabas y fonemas (actividades de identificación, adición y omisión), con un total de 30 ítems (15 de sílabas y 15 de fonemas). La puntuación máxima que puede obtenerse es 30 , un punto por cada respuesta correcta y cero por cada error. La confiabilidad, medida a través del coeficiente alfa de Cronbach es de .80 .

- Batería de inicio a la lectura (BIL): Se utilizó la batería de inicio a la lectura elaborada por Sellés, Martínez, Vidal-Abarca y Gilabert (2008) con la finalidad de evaluar las habilidades que facilitan el acceso a la lectura, en concreto se utilizaron las subpruebas: Reconocer palabras, reconocer frases, funciones de la lectura y conocimiento del nombre de las letras. La puntuación en cada una de estas pruebas se obtiene asignando un punto a cada respuesta correcta. Estos subtest presentan un coeficiente de fiabilidad de Cronbach de 0.78.

- Velocidad de nombrado. The Rapid Automatized Naming Test (RAN) (Wolf \& Denckla, 2003): El objetivo de la tarea es nombrar 200 estímulos lo más rápido posible, agrupados en cuatro subtests; dígitos, letras, colores y dibujos. En el registro de la tarea RAN se anota el tiempo que se tarda en nombrar los estímulos de cada cartulina y el número de errores que se cometen al nombrarlos. Con estos dos datos se realiza un índice de eficiencia por cada uno de los 4 tipos de subtests presentados, según el procedimiento utilizado por Compton (2003) que consiste en convertir las puntuaciones en dígitos por segundo, letras por segundo, colores por segundo y dibujos por segundo. Esta prueba presenta un coeficiente de fiabilidad de Cronbach de 0.80 .

- Evaluación de los procesos de lectura: Para la evaluación de la lectura se han utilizado cuatro subtest del test PROLEC-R (Cuetos, Rodríguez, Ruano \& Arribas, 2007). Se emplearon las pruebas de lectura de palabras y pseudopalabras para valorar los procesos léxicos y los subtest de estructuras gramaticales y comprensión de oraciones para evaluar los procesos semánticos. La puntuación total en cada una de estas cuatro pruebas se obtiene asignando un punto a cada respuesta correcta, además en las dos primeras se tiene en consideración el tiempo invertido en cada subtest. Esta prueba presenta un coeficiente de fiabilidad de Cronbach de 0.79 . 


\subsection{Procedimiento de recogida y análisis de datos}

El trabajo utilizó un diseño cuasi-experimental de medidas repetidas pretestpostest con grupo control. El análisis de la potencia estadística permite la selección de un tamaño muestral que garantice el éxito de la investigación (Davey \& Savla, 2009), presentando la muestra en este estudio un valor de 0.912 .

Antes y después de implementar el plan de intervención se aplicaron cuatro instrumentos de evaluación a los alumnos experimentales y control con el propósito de medir las variables dependientes sobre las que se hipotetizó que el programa iba a tener efecto: Conciencia fonológica, velocidad de denominación y conocimiento alfabético. La aplicación de las pruebas antes y después de implementar el programa se llevó a cabo por profesionales de la educación (docentes especialistas en Audición y Lenguaje y psicopedagogos) previamente entrenados, lo que facilitó la homogeneidad en la recogida de los datos.

La valoración inicial de los alumnos se realizó de forma individual en espacios próximos al aula ordinaria en el mes de octubre y dentro del horario escolar. Posteriormente se implementó el plan de intervención en los grupos experimentales (60 sesiones distribuidas en 5 clases de 45 minutos a la semana), el mismo tiempo que los grupos control siguieron el programa de aprendizaje de la lectura mediante el libro de texto establecido. En el último trimestre del curso se volvió a realizar la evaluación a todo el alumnado con los mismos instrumentos. El estudio respetó los valores éticos requeridos en la investigación con seres humanos (consentimiento informado, protección de datos personales, no discriminación, gratuidad y tener la posibilidad de abandonar el programa en cualquiera de sus fases).

\subsection{Programa de intervención}

El programa de intervención que se utilizó tenía como objetivo desarrollar de manera explícita la conciencia fonológica, la velocidad de denominación y el conocimiento alfabético.

La conciencia fonológica se trabajó con diferentes tareas de segmentación léxica, conciencia silábica y conciencia fonémica mediante propuestas de carácter lúdico a través de actividades de: Identificación, comparación, clasificación, sustitución y omisión de sílabas y fonemas. Para el desarrollo de la segmentación léxica se llevaron a cabo tareas centradas en: Reconocer y comparar palabras por su longitud, dividir oraciones compuestas de varias palabras, elaborar oraciones a partir de una serie de palabras dadas y componer oraciones según un número de palabras establecido. La conciencia silábica se trabajó mediante actividades de reconocimiento del número de sílabas en diferentes palabras, identificar palabras atendiendo a la sílaba inicial y final, sustituir sílabas en las palabras para formar otras nuevas, añadir sílabas en posición inicial y final. La conciencia fonémica se ejercitó a través de tareas centradas en 
recomponer palabras a partir de la síntesis fonémica, identificar palabras atendiendo al fonema inicial y final, sustituir y omitir fonemas en las palabras en distintas posiciones. Inicialmente se abordó el desarrollo de las habilidades de conciencia léxica y silábica y posteriormente se ejercitó la conciencia fonémica siguiendo la secuenciación y temporalización presentada en los materiales Avanza (Espejo, Gutiérrez, Llambés \& Vallejo, 2008) y Avanzados (Espejo, Gutiérrez, Llambés \& Vallejo, 2015).

El programa para el aprendizaje del conocimiento alfabético que se utilizó estaba centrado en la enseñanza del nombre de las letras mediante métodos mixtos de base fonética. Se presentaban las distintas grafías junto con palabras del entorno del alumnado, las cuales se trabajaban a nivel visual, auditivo, táctil y articulatorio, tanto de manera aislada como dentro de palabras del entorno del niño y de títulos de cuentos infantiles. Dichos cuentos posteriormente se leían de manera conjunta mediante la técnica de la lectura dialógica, caracterizada por simultanear la lectura en voz alta por parte del profesor con situaciones interactivas como el diálogo y la formulación de preguntas.

La velocidad de denominación se trabajó mediante plantillas de diferentes imágenes: Números, letras, colores y objetos relacionados con la temática de los cuentos trabajados. Se presentaban en la pizarra digital al finalizar la lectura conjunta del cuento para ser evocados con agilidad por los alumnos de izquierda a derecha, misma dirección que se realiza durante la lectura. Se combinaba la lectura del profesor con la lectura por parte de los distintos alumnos, tanto de manera individual, en pequeño grupo, como de manera colectiva.

\section{Resultados}

Con la finalidad de analizar el cambio en las variables objeto de estudio se realizaron análisis descriptivos (medias y desviaciones típicas) con los datos obtenidos en los test administrados en la fase pretest, postest y en la diferencia postest-pretest, además de análisis de varianza con las puntuaciones pretest (MANOVAs, ANOVAs) y análisis de covarianza (MANCOVAs, ANCOVAs) de las diferencias encontradas postest-pretest en experimentales y control en las variables medidas antes y después de la intervención. Dichos análisis se realizaron con el programa SPSS 20.0. Además, se calculó el tamaño del efecto ( $\mathrm{d}$ de Cohen) (pequeño $<.50$; moderado .50-.79; grande $\geq$.80). Los resultados del MANOVA pretest para el conjunto de variables puso de relieve que antes de la intervención no existían diferencias significativas entre experimentales y control, $F(1,56)=1.43, p>.05$. Sin embargo, los resultados del MANCOVA de las diferencias postest-pretest, utilizando las puntuaciones pretest como covariables fueron significativos $F(1,56)=2.67, p<.05$. Estos datos indican que el programa de intervención tuvo un efecto significativo. Para analizar el cambio en cada 
variable se realizaron análisis descriptivos y de varianza los cuales se presentan en la Tabla 1.

Tabla 1. Medias y Desviaciones Típicas en conciencia fonológica, velocidad de denominación y conocimiento alfabético y resultados del análisis de varianza y covarianza para el grupo experimental y control.

\begin{tabular}{|c|c|c|c|c|c|c|c|c|c|c|c|c|c|c|c|c|c|}
\hline \multirow{4}{*}{\begin{tabular}{|l} 
\\
Variables \\
\end{tabular}} & \multirow{2}{*}{\multicolumn{6}{|c|}{$\begin{array}{l}\text { Grupo Experimental } \\
\qquad(\mathrm{n}=206)\end{array}$}} & \multirow{2}{*}{\multicolumn{6}{|c|}{$\begin{array}{l}\text { Grupo Control } \\
\qquad(\mathrm{n}=202)\end{array}$}} & \multicolumn{5}{|c|}{$\begin{array}{l}\text { Experimental - Control } \\
\qquad(\mathrm{n}=408)\end{array}$} \\
\hline & & & & & & & & & & & & & \multirow{2}{*}{\multicolumn{2}{|c|}{$\begin{array}{c}\text { Anova } \\
\text { Pretest }\end{array}$}} & \multirow{3}{*}{$\begin{array}{c}\text { Anova } \\
\text { Postest-Pretest } \\
\mathrm{F}(1,56)\end{array}$} & \multirow{2}{*}{\multicolumn{2}{|c|}{$\begin{array}{c}\text { Ancova } \\
\text { Postest-Pretest }\end{array}$}} \\
\hline & \multicolumn{2}{|c|}{ Pre } & \multicolumn{2}{|c|}{ Post } & \multicolumn{2}{|c|}{ Post-Pre } & \multicolumn{2}{|c|}{ Pre } & \multicolumn{2}{|c|}{ Post } & \multicolumn{2}{|c|}{ Post-Pre } & & & & & \\
\hline & M & DT & $\mathrm{M}$ & DT & $\mathrm{M}$ & DT & M & DT & M & DT & M & DT & $\mathrm{F}(1,56)$ & $\mathrm{d}$ & & & d \\
\hline PECO & & & & & & & & & & & & & & & & & \\
\hline C. Silábica & 2.83 & .56 & 3.80 & .74 & .97 & .52 & 2.92 & .62 & 3.75 & .34 & .83 & .62 & 1.56 & .23 & .435 & 1.25 & .24 \\
\hline C. Fonémica & 2.67 & .83 & 3.88 & .82 & 1.21 & .61 & 2.71 & .46 & 2.94 & .82 & .23 & .83 & .72 & .45 & $6.46^{* * * *}$ & $6.78^{* * * *}$ & .52 \\
\hline RAN & & & & & & & & & & & & & & & & & \\
\hline Números & 1.96 & .64 & 2.48 & .43 & .52 & .31 & 2.03 & .62 & 2.46 & .62 & .43 & .36 & 2.53 & .22 & 4.63 & 3.35 & .26 \\
\hline Letras & 1.98 & .45 & 2.86 & .71 & .88 & .55 & 2.05 & .33 & 2.32 & .54 & .27 & .51 & 1.24 & .43 & $2.54 * *$ & $3.65^{* *}$ & .46 \\
\hline Colores & 2.04 & .52 & 2.75 & .43 & .71 & .43 & 2.10 & .55 & 2.48 & .45 & .38 & .93 & 1.61 & .53 & $7.04 * *$ & $6.47 * *$ & .56 \\
\hline Dibujos & 2.03 & .31 & 2.52 & .63 & .49 & .82 & 2.07 & .37 & 2.30 & .37 & .23 & .52 & 3.82 & .23 & 1.36 & .265 & .24 \\
\hline BIL & & & & & & & & & & & & & & & & & \\
\hline Reconocer palabras & 1.96 & .56 & 2.59 & .72 & .65 & .54 & 2.05 & .40 & 2.29 & .63 & .24 & .22 & 2.57 & .25 & $3.46^{* *}$ & $4.32 * *$ & .34 \\
\hline Reconocer frases & 1.88 & .53 & 2.61 & .52 & .83 & .63 & 1.97 & .35 & 2.38 & .34 & .41 & .53 & 1.23 & .32 & $2.64 * *$ & $2.46^{* *}$ & .33 \\
\hline Funciones de la lectura & 2.02 & .46 & 2.56 & .61 & .54 & .34 & 2.06 & .72 & 2.58 & .67 & .52 & .68 & 1.42 & .27 & 1.42 & .262 & .31 \\
\hline C. Alfabético & 2.01 & .71 & 2.73 & .58 & .72 & .67 & 2.02 & .47 & 2.27 & .58 & .25 & .43 & .731 & .46 & $5.03 * * *$ & $6.41^{* * * *}$ & .52 \\
\hline PROLEC-R & & & & & & & & & & & & & & & & & \\
\hline Lectura palabras & 1.87 & .73 & 2.63 & .41 & .76 & .72 & 1.88 & .57 & 2.05 & .84 & .17 & .56 & .48 & .42 & $7.24 * * *$ & $8.46^{* * * *}$ & .56 \\
\hline Lectura pseudopalabras & 1.52 & .54 & 2.15 & .64 & .63 & .56 & 1.56 & .81 & 1.72 & .66 & .16 & .79 & .52 & .35 & $6.52 * * *$ & $9.46^{* * *}$ & .37 \\
\hline E. gramaticales & 1.36 & .65 & 2.06 & .81 & .73 & .52 & 1.42 & .53 & 1.53 & .59 & .11 & .95 & .49 & .41 & $9.73^{* * * *}$ & $12.43 * * *$ & .51 \\
\hline C. oraciones & 1.42 & .43 & 2.14 & .76 & .72 & .63 & 1.50 & .64 & 1.75 & .74 & .25 & .73 & .37 & .45 & $8.37 * * *$ & $9.54 * * *$ & .43 \\
\hline
\end{tabular}

\section{Cambios en la conciencia fonológica}

Con el objeto de analizar la eficacia del programa en el desarrollo de la conciencia fonológica, se estudiaron los cambios en las puntuaciones obtenidas en el Test PECO. El MANOVA pretest no evidenció diferencias significativas entre experimentales y control, $F(1,56)=2.57, p>.05$, sin embargo, los resultados del MANCOVA postestpretest, $F(1,56)=2.82, p<.05$, confirmaron diferencias de carácter significativo entre ambas condiciones. En el análisis de cada variable de forma independiente únicamente se ratificaron diferencias en la 'conciencia fonémica', en la que se observó un aumento mayor en los experimentales $(M=1.21)$ que en los del grupo control $(M=.23)$. Los resultados del ANOVA pretest pusieron de manifiesto que en esta fase no había diferencias significativas entre experimentales y control, $F(1,56)=.72, p>.05$, sin embargo, el ANCOVA postest-pretest puso de relieve diferencias estadísticamente significativas entre condiciones, $F(1,56)=6.78, p<.01$. El tamaño del efecto fue moderado $(r=.52)$. Lo que indica una mejora de la capacidad para tomar conciencia de las unidades mínimas de las palabras atribuible al programa de intervención.

\section{Cambios en la velocidad de denominación}

Para analizar la eficacia del programa en el desarrollo de la velocidad de denominación, se estudiaron los cambios en las puntuaciones obtenidas en el Test RAN. El MANOVA pretest realizado con el conjunto de las cuatro variables medidas (nombrar números, letras, colores y dibujos) no evidenció la presencia de diferencias significativas en la fase pretest entre experimentales y control, $F(1,56)=2.24, p>.05$. Sin 
embargo, se encontraron diferencias significativas en el MANOVA postest-pretest, $F(1,56)=3.14, p<.01$, al igual que en el MANCOVA postest-pretest, $F(1,56)=2.43$, $p<.01$. Como se puede observar en la Tabla 1, en la variable 'denominación de letras', la muestra experimental obtiene un incremento $(M=.88)$, superior al conseguido por el grupo control $(M=.27)$. Los resultados del ANOVA pretest pusieron de manifiesto que en esta fase no había diferencias significativas entre experimentales y control, $F(1,56)=1.24, p>.05$. Sin embargo, los datos del ANCOVA de las diferencias postestpretest evidenció resultados significativos, $F(1,56)=3.65$, $p<.01$. El tamaño del efecto fue pequeño $(r=.46)$.

En la variable 'denominación de colores' se constatan de igual modo incrementos superiores en los experimentales $(M=.71)$ frente a los del grupo control $(M=.38)$. Los resultados del ANOVA pretest evidenciaron que a priori no existían diferencias significativas entre ambas condiciones, $F(1,56)=1.61, p<.05$, realizándose un ANCOVA de las diferencias postest-pretest que indicó diferencias significativas, $F(1,56)=6.47, p<.01$, siendo el tamaño del efecto moderado $(\mathrm{r}=.56)$. Lo que pone de manifiesto un incremento en la velocidad de denominación como consecuencia de la realización del programa de intervención.

\section{Cambios en el reconocimiento de las unidades lingüisticas}

Con el propósito de evaluar el impacto del programa en las habilidades facilitadoras de la lectura, se analizaron los cambios en los resultados obtenidos en el Test BIL. El MANOVA pretest realizado con el conjunto de las cuatro variables medidas (reconocer palabras, reconocer frases, funciones de la lectura y conocimiento alfabético) no evidenció la presencia de diferencias significativas en la fase pretest entre experimentales y control, $F(1,56)=3.15, p>.05$, pero tampoco se encontraron diferencias en el MANCOVA postest-pretest $F(1,56)=3.47, p>.05$. En el análisis de cada variable de forma independiente se produjeron diferencias en el 'reconocimiento de palabras', el grupo experimental obtiene una mejora $(\mathrm{M}=.65)$, mayor que la conseguida por el grupo control $(M=.24)$. Los resultados del ANOVA pretest pusieron de manifiesto que en esta fase no existían diferencias que fueran significativas entre experimentales y control, $F(1,56)=2.57, p>.05$. Sin embargo, los datos del ANCOVA de las diferencias postest-pretest indicaron resultados significativos, $F(1,56)=4.32, p<.01$. El tamaño del efecto fue pequeño $(r=.34)$. En la variable 'reconocimiento de frases' se constatan de igual modo incrementos superiores en los experimentales $(M=.83)$ frente a los del grupo control $(M=.41)$. En el ANOVA pretest los datos obtenidos pusieron de manifiesto que en esta fase no había diferencias significativas entre experimentales y control, $F(1,56)=1.23, p>.05$. No obstante, los datos del ANCOVA de las diferencias postest-pretest indicaron resultados significativos, $F(1,56)=2.46, p<.01$. El tamaño del efecto fue pequeño $(\mathrm{r}$ $=.33$ ). También se produjo una mejora tendencialmente significativa en 'conocimiento 
alfabético', con un aumento mayor en los experimentales $(M=.72)$ que en el alumnado perteneciente al grupo control $(M=.25)$. Los resultados del ANOVA pretest pusieron de manifiesto que en esta fase no había diferencias significativas entre experimentales y control, $F(1,56)=.731, p>.05$. Sin embargo, los datos del ANCOVA de las diferencias postest-pretest indicaron resultados significativos, $F(1,56)=6.41, p<.001$. El tamaño del efecto fue moderado $(\mathrm{r}=.52)$. Estos datos ponen de manifiesto una mejora del aprendizaje del conocimiento alfabético y de los componentes del lenguaje escrito atribuible al programa de intervención puesto en práctica.

\section{Cambios en la decodificación de palabras escritas}

Para evaluar si el programa fue eficaz en la decodificación de las palabras que intervienen en el aprendizaje de la lectura se analizaron los cambios en las puntuaciones logradas en el Test PROLEC-R. El MANOVA pretest realizado para el conjunto de las variables de la prueba puso de manifiesto que no existían diferencias significativas en la fase pretest entre experimentales y control, $F(1,56)=3.53, p>.05$. Sin embargo, se encontraron diferencias significativas en el MANOVA postest-pretest, $F(1,56)=2.46, p<.01$, al igual que en el MANCOVA postest-pretest, $F(1,56)=3.67$, $p<.01$. Como se puede observar en la Tabla 1, en la variable 'lectura de palabras' el grupo experimental obtiene una mejora $(M=.76)$, mayor que la conseguida por el grupo control $(M=.17)$. Los resultados del ANOVA pretest pusieron de manifiesto que en esta fase no existían diferencias que fueran significativas entre experimentales y control, $F(1,56)=.48, p>.05$. Sin embargo, los datos del ANCOVA de las diferencias postest-pretest indicaron resultados significativos, $F(1,56)=8.46, p<.001$. El tamaño del efecto fue moderado $(r=.56)$. Los resultados del ANOVA pretest en la 'lectura de pseudopalabras' no evidencian diferencias entre experimentales y control, $F(1,56)=.52$, $p>.05$, sin embargo, los resultados del ANCOVA postest-pretest, $F(1,56)=9.46$, $p<.001$, fueron significativos. Como se puede observar se produce un aumento superior en los experimentales $(M=.63)$ frente a los de control $(M=.16)$. El tamaño del efecto fue pequeño $(\mathrm{r}=.37)$. La misma dirección se observa en 'estructuras gramaticales' en las que el ANOVA pretest no evidencia diferencias entre experimentales y control, $F(1,56)=.49, p>.05$, sin embargo, los resultados del ANCOVA postest-pretest, $F(1,56)=12,43, \quad p<.001$, fueron significativos. Los experimentales aumentaron significativamente la comprensión de estructuras gramaticales $(M=.73)$ frente a los de control $(M=.11)$. El tamaño del efecto fue moderado $(\mathrm{r}=.51)$. Por último, en 'comprensión de oraciones' el ANOVA pretest puso de relieve que no había diferencias significativas entre experimentales y control, $F(1,56)=.37, p>.05$, sin embargo, los resultados del ANCOVA pretest-postest, $F(1,56)=9.54, p<.001$, evidenciaron diferencias significativas entre ambas condiciones. Como se presenta en la Tabla 1, los experimentales aumentaron significativamente la comprensión de oraciones $(M=.72)$ frente a los de control $(M=.25)$. El tamaño del 
efecto fue pequeño $(\mathrm{r}=.43)$. Estos datos ponen de manifiesto una mejora del aprendizaje de la lectura atribuible al programa de intervención puesto en práctica.

\section{Influencia del género en los efectos del programa}

Con la finalidad de explorar si el programa tuvo un efecto diferencial con respecto al sexo, es decir, si fue más beneficiosa para los chicos o las chicas, o si el cambio producido por el programa era similar en ambos sexos se efectuaron análisis descriptivos (medias y desviaciones típicas) y de varianza (ANOVAs, ANCOVAs) en la fase de pretest y en las diferencias postest-pretest en función del género. Los resultados se exponen en la Tabla 2.

Tabla 2. Medias y Desviaciones Típicas de los participantes experimentales en conciencia fonológica, velocidad de denominación y conocimiento alfabético en función del género, en la fase pretest y en la diferencia postest-pretest.

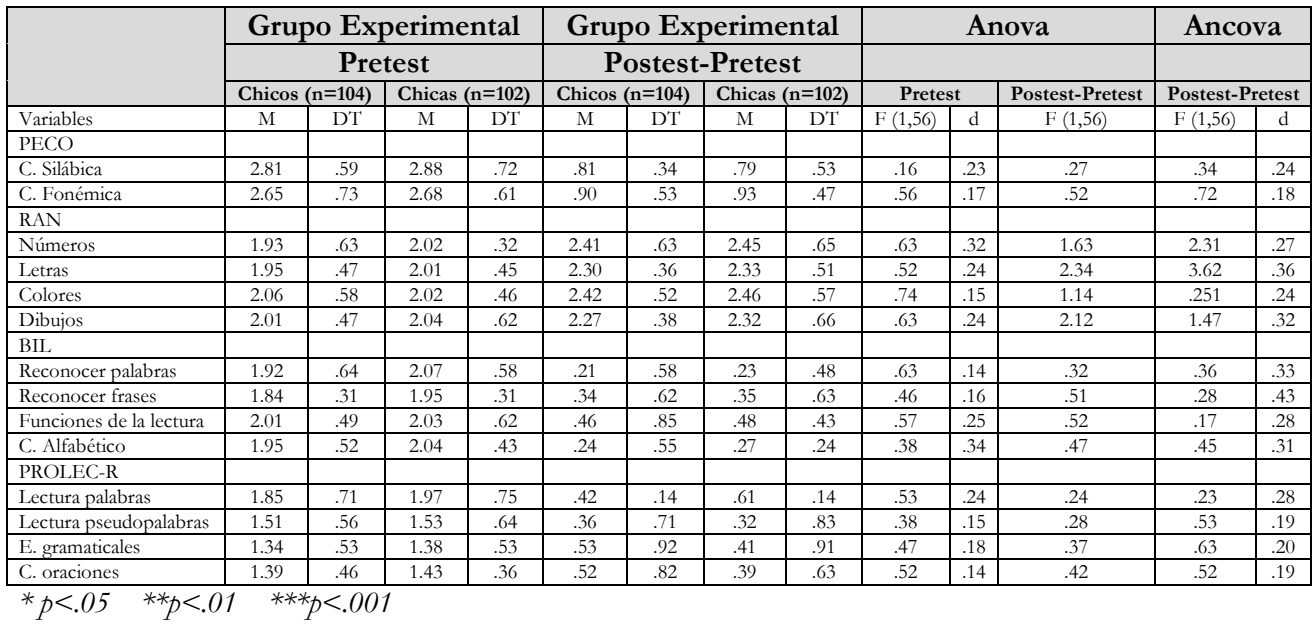

En relación al desarrollo de la conciencia fonológica, ni el MANCOVA postestpretest, $F(1,56)=3.27, p>.05$, ni los ANCOVAS postest-pretest (ver Tabla 2) mostraron diferencias significativas en función del género. En el desarrollo léxico, el MANCOVA postest-pretest $F(1,56)=2.73$, $p>.05$, ni los ANCOVAS postest-pretest mostraron diferencias significativas. En la velocidad de denominación, el MANCOVA postest-pretest $F(1,56)=3.14, p>.05$, ni los ANCOVAS postest-pretest mostraron diferencias significativas. En las habilidades favorecedoras de la lectura, el MANCOVA postest-pretest $F(1,56)=2.46, p>.05$, ni los ANCOVAS postest-pretest mostraron diferencias significativas. De igual modo, respecto a las variables relativas a los procesos implicados en la lectura ni el MANCOVA postest-prestest $F(1,56)=3.52$, $p>.05$, ni los ANCOVAS postest-pretest evidenciaron diferencias.

En consecuencia, en ninguna de las variables estudiadas se produjeron efectos diferenciales del programa en función del género. 


\section{CONCLUSIONES}

El objetivo de este estudio era comprobar el efecto que la intervención conjunta en las variables de: Conciencia fonológica, velocidad de denominación y conocimiento alfabético, presentan en la mejora de la adquisición de la lectura. Los resultados obtenidos ponen de manifiesto que la instrucción sobre estas variables mejora de manera significativa el proceso de adquisición de la lectura. Estos datos amplían los aportes que se han venido efectuando recientemente sobre las relaciones entre conciencia fonológica y velocidad de denominación respecto al aprendizaje de la lectura, ya que más allá del propósito de otros estudios centrados en identificar las correlaciones entre estas variables (Rabazo, et al., 2016) en el presente trabajo se esclarecen a modo de causa-efecto las vinculaciones existentes entre los principales predictores de la lectura, lo cual tiene implicaciones directas tanto para el diseño de los programas orientados al aprendizaje de la lectura como para el tratamiento e intervención de los alumnos con dificultades en esta habilidad lingüística.

Analizando los diferentes componentes que intervienen en la facilitación del proceso de aprendizaje de la lectura, se comprueba que el grupo experimental ha mejorado de manera significativa respecto al grupo control en el procesamiento fonológico, aunque si bien, no todos los niveles del conocimiento fonológico presentan el mismo grado de importancia en el aprendizaje de la lectura. Los datos recogidos indican que es la toma de conciencia de las unidades mínimas del habla la que favorece en mayor medida este aprendizaje mientras que la conciencia silábica no presenta la misma relevancia. Este hecho coincide con los hallazgos de otros trabajos en los que se pone de manifiesto la influencia que presentan los distintos niveles de conciencia fonológica en el aprendizaje de la lectura (Arancibia, et al., 2012; Rabazo, et al., 2016). De donde se deduce que los aprendices a medida que progresan en el conocimiento metalingüístico son más sensibles a las unidades más pequeñas de la palabra, inicialmente toman conciencia de las sílabas y posteriormente de los fonemas, siendo estos últimos los elementos que más favorecen el proceso de adquisición de la lectura.

También el alumnado del grupo experimental logró resultados mayores en la velocidad para identificar y evocar eficazmente diferentes elementos tanto lingüísticos como no lingüísticos, especialmente letras y colores. Se interpreta que la intervención en velocidad de denominación mejora el conocimiento ortográfico y la memoria de palabras con regularidad ortográfica lo que influye en la lectura de un gran número de palabras de manera global dando lugar a una mejor exactitud lectora. Estos datos coinciden con los aportes de López-Escribano, Sánchez-Hípola, Suro y Leal (2014) quienes señalan que la velocidad de denominación es un potente indicador tanto para predecir la adquisición posterior de la lectura desde las primeras edades, como para discriminar entre lectores típicos y otros con dificultades. Estos resultados coinciden con los aportes de otros estudios (Gómez-Velázquez, et al., 2014; González, Cuetos, 
Vilar \& Uceira, 2015) que indican que la velocidad de denominación constituye una habilidad importante en el proceso alfabetización inicial en las primeras edades, ofreciéndose en este trabajo una mayor concreción sobre los factores que influyen en mayor medida en la automatización de las relaciones grafía-sonido y en el procesamiento ortográfico.

Respecto a la capacidad predictiva tanto de la conciencia fonológica como de la velocidad de denominación, existen evidencias de que ambas intervienen de manera diferente en el aprendizaje lector, así mientras que el procesamiento fonológico tiene mayor relación con la exactitud lectora en cuanto que ofrece herramientas para el deletreo temprano, desarrolla el reconocimiento de palabras permitiendo la segmentación en subunidades lingüísticas y favorece la comprensión lectora, la velocidad de denominación presenta una influencia más elevada sobre la fluidez lectora al facilitar el acceso a las representaciones ortográficas de las palabras que están almacenadas en la memoria a largo plazo (González et al., 2015). Lo que evidencia la conveniencia de incluir en los programas didácticos orientados a favorecer el aprendizaje lector, el desarrollo conjunto de ambas facetas.

En relación a la adquisición de las habilidades favorecedoras de la lectura, los datos obtenidos en este estudio señalan que los alumnos pertenecientes al grupo experimental logran resultados superiores al control en la capacidad para reconocer palabras, frases y en el conocimiento alfabético, lo que indica que la enseñanza del código lingüístico a través de elementos del entorno próximo y significativo como los nombres de los compañeros de clase, los personajes y los títulos de cuentos infantiles potencian la identificación y el reconocimiento del principio alfabético desde los primeros momentos en los que se accede al aprendizaje del código escrito, máxime cuando se ponen en práctica modelos de 'lectura dialógica'. Estos datos coinciden con los aportes de otros estudios en los que se ha demostrado el efecto positivo del aprendizaje compartido en el proceso de adquisición de la lectura (Gutiérrez, 2016b).

Respecto a los procesos cognitivos de aprendizaje de la lectura, los datos obtenidos señalan que el programa de intervención contribuyó de manera relevante a la mejora de las distintas variables implicadas en la lectura, tanto de palabras como de 'pseudopalabras', lo que evidencia que los alumnos participantes en el programa logran una mejoría en el procesamiento fonológico y ortográfico. Este logro en la automatización de las reglas de correspondencia fonema-grafema puede venir determinado por el desarrollo de las habilidades de conciencia fonológica, así como por el incremento en la capacidad para visualizar y recuperar de forma rápida y precisa la representación fonológica de las palabras.

Respecto al género, no se encontraron diferencias en el aprendizaje lector, ni en relación al proceso decodificador, lo que cuestiona la necesidad de desarrollar didácticas diferenciales según el género. 
En resumen, este trabajo contribuye a la facilitación de los procesos de aprendizaje de la lectura, permitiendo orientar el diseño y la realización de actividades educativas que incidan en la mejora del aprendizaje de la lectura, así como en la prevención sobre las dificultades de esta habilidad lingǘstica, por lo que se recomienda su implementación en el alumnado que se inicia en el aprendizaje lector así como con el alumnado perteneciente al grupo control de esta investigación ya que no se benefició de los efectos del programa.

En consecuencia, a partir de los resultados de este estudio y a tenor de las repercusiones negativas que las dificultades de lectura presentan en el aprendizaje escolar, a nivel práctico se sugiere el diseño de programas orientados al desarrollo de los procesos de aprendizaje de la lectura mediante propuestas didácticas basadas en el desarrollo de la conciencia fonológica, especialmente mediante tareas centradas en la toma de conciencia de las unidades mínimas del habla, junto con actividades que fomenten la velocidad de denominación en concreto de letras y colores. De igual modo, se recomienda la puesta en práctica de dinámicas de lectura dialógica en las que se potencie el análisis reflexivo, el pensamiento conjunto en voz alta, la participación activa del aprendiz, así como la posibilidad de hacer explícitas las estrategias e hipótesis que los propios alumnos van realizando sobre el proceso de adquisición de la lectura. Una limitación de este trabajo y que sería interesante considerar en futuros estudios es que no se atendió a determinadas variables a las que los niños se hallan expuestos, como son las prácticas de lectura compartida en el ámbito familiar, el ambiente de lectura en el hogar o el desarrollo léxico, que de igual modo pueden resultar determinantes en el proceso inicial del aprendizaje de la lectura.

\section{REFERENCIAS BIBLIOGRÁFICAS}

Arancibia, B., Bizama, M. \& Sáez, K. (2012). Aplicación de un programa de estimulación de la conciencia fonológica en preescolares y alumnos de primer año básico pertenecientes a escuelas vulnerables de la Provincia de Concepción, Chile. Revista Signos. Estudios de Lingüistica, 45(80), 236-256.

Compton, D. L. (2003). Modeling the relationship between growth in rapid naming speed and growth in decoding skill in first-grade children. Journal of Educational Psychology, 95, 225-239.

Cuetos, F., Rodríguez, B., Ruano, E. \& Arribas, D. (2007). Prolec-R, Batería de evaluación de los procesos lectores, Revisada. Madrid: TEA.

Davey, A. \& Savla, J. (2009). Estimating statistical power with incomplete data. Organizational Research Methods, 12(2), 320-346.

De Jong, P. F. \& Van der Leij, A. (2002). Effects of phonological abilities and linguistic comprehension on the development of reading. Scientific Studies of Reading, 6, 51-77. 
Diuk, B. \& Ferroni, M. (2012). Dificultades de lectura en contextos de pobreza: ¿Un caso de Efecto Mateo? Psicología Escolar e Educacional, 16(2), 209-217.

Espejo, P., Gutiérrez, R., Llambés, D. \& Vallejo, B. (2008). Avanza: Programa para el desarrollo de las habilidades escolares básicas. Habilidades fonológicas I. Alicante: ECU.

Espejo, P., Gutiérrez, R., Llambés, D. \& Vallejo, B. (2015). Avanzados: Programa para el desarrollo de las habilidades escolares básicas. Habilidades fonológicas II. Alicante: ECU.

Gómez-Velázquez, F. R., González-Garrido, A. A., Matute, E. \& Ramos-Loyo, J. (2014). Relación entre la velocidad de denominación, el procesamiento auditivo temporal de orden y la adquisición de la lectura. Revista Neuropsicología, Neuropsiquiatría y Neurociencias 14(1), 249-267.

González, R. M., Cuetos, F., Vilar, J. \& Uceira, E. (2015). Efectos de la intervención en conciencia fonológica y velocidad de denominación sobre el aprendizaje de la escritura. Aula Abierta, 43, 1 - 8.

González, R. M., López, S., Vilar J. \& Rodríguez, A. (2013). Estudio de los predictores de la lectura. Revista de investigación en educación, 11(2), 98-110.

Gutiérrez, R. (2016a). Habilidades favorecedoras del aprendizaje de la lectura en las primeras edades. En actas del VIII Congreso Internacional de Psicología y Educación (pp. 1385-1386). Asociación Científica de Psicología y Educación: Toledo.

Gutiérrez, R. (2016b). Efectos de la lectura dialógica en la mejora de la comprensión lectora en alumnos de Educación Primaria. Revista de Psicodidáctica, 21(2), 303320 .

Kim Y-S. \& Pallante, D. (2012). Predictors of reading skills for kindergartners and first grade students in Spanish: A longitudinal study. Reading and Writing, 25, 122.

López-Escribano, C. \& Beltrán, J. (2009). Early predictors of reading in three groups of native Spanish speakers: Spaniards, Gypsies, and Latin Americans. The Spanish Journal of Psychology, 12, 84-95.

López-Escribano, C., Sánchez-Hípola, P., Suro, J. \& Leal, F. (2014). Análisis comparativo de estudios sobre la velocidad de nombrar en español y su relación con la adquisición de la lectura y sus dificultades. Universitas Psychologica, 13(2), 757-769.

Martínez, J. M. (2014). Relación entre funciones ejecutivas, conciencia fonológica y lectura inicial, en el alumnado del $1^{\circ}$ curso de Educación Primaria. Revista educación y futuro digital, 10, 65-80. 
Rabazo, M. J., García, M. \& Sánchez, S. (2016). Exploración de la conciencia fonológica y la velocidad de nombrado en alumnos de $3^{\circ}$ Educación Infantil y $1^{\circ}$ de Educación Primaria y su relación con el aprendizaje de la lectoescritura. Revista de Psicología, 1(1), 83-94.

Ramos, J. L. \& Cuadrado, I. (2006). Prueba para la Evaluación del Conocimiento Fonológico. PECO. Madrid: EOS.

Sellés, P., Martínez, T., Vidal-Abarca. E. \& Gilabert, R. (2008). BIL 3-6. Batería de Inicio a la lectura. Madrid: ICCE.

Suárez-Coalla, P., García de Castro, M. \& Cuetos, F. (2013). Variables predictoras de la lectura y la escritura en castellano. Infancia y aprendizaje, 36(1), 77-89.

Wolf, M. \& Denckla, M. (2003). Rapid automatized naming tests. Greenville, SC: Super Duper.

Wolf, M., O’Rourke, A. G., Gidney, C., Lovett, M., Cirino, P. \& Morris, R. (2002). The second deficit: An investigation of the independence of phonological and naming-speed deficits in developmental dyslexia. Reading and Writing, 15, 4372. 